\title{
Research on the Strategy of Group Vehicle Intelligent Perception and Traffic Route Guidance of Semantic Car Road Network
}

\author{
L.J. Tai, R.F. Hu, C.W. Chen \\ School of mechanical engineering \\ Ningbo University of Technology \\ Ningbo, China
}

\begin{abstract}
Vehicle road network has become the development direction of the future transport system. In this paper, semantic modeling of car road network is built, semantic integration and sharing problems of heterogeneous networking information is solved. The Group car intelligent perception and effective cognitive method is explored. The dynamic traffic flow guidance strategies is proposed, to realize intelligent processing of massive car road network information.
\end{abstract}

Keywords-car road network; traffic route guidance; intelligent transportation system

\section{INTRODUCTION}

With the high speed development of economy and the improvement of people's living standard, in recent years our country automobile industry showed leap type development. In the end of 2013, car ownership reach 137000000 vehicles, forecast it will reach 200000000 vehicles in 2020.But the attendant problem of traffic congestion, traffic accidents, environmental pollution and energy shortage has become a common problem the world facing.

Transportation, automobile manufacturing and other traditional separation industry is moving toward integrating with information communication, with the progress and application of the Internet, the Internet of things, cloud computing, data technology. At present, China's automobile industry developing at a surprising speed, with vigorously push forward of this emerging industry the Internet of things in our country the 12th five-year plan, make the intelligent transportation system of vehicle road network has become the development direction of the future transport system.

Vehicle road network is one of the most effective industry that promote the industrialization deply fuse information. Vehicle road network industry influence surface is very wide, value spread widely, led and radiation ability is very strong , from the automobile consumer services, automotive design and manufacturing, vehicle maintenance to finance and insurance, public transportation management, logistics and transport, urban public management etc. In addition, the development of vehicle road network will also improve the basic contradiction of social traffic, Including the contradiction between personal travel demand and traffic efficiency, the contradiction between travel safety and public traffic safety, the contradiction between energy saving and carbon emission. Therefore, the vehicle road network technology is practical meaningful to study on the development of China's automobile industry.

This paper will carry on deeply research on semantic modeling, intelligent information perception and traffic route guidance on issues such as road of vehicle road network on the basis of previous studies. To provide guidance for the development of intelligent transportation system of China.

\section{SEmantic Modeling OF CAR RoAd NETWORK}

The heterogeneous of vehicle road network information must be solved firstly to complete the vehicle road network information integration. Ontology is an effective technology to realize semantic integration. If the ontology method is introduced in the knowledge expression of car road network, the recognized or default or implicit knowledge of car road network can be expressed by a display, and formal way, complete semantic integration of vehicle road network, realize knowledge semantic sharing of vehicle road network intelligent traffic control system .

Definition method of ontology in the field of car networking is descripted as follows.

\section{A. Definition}

Ontology of car network is a formal description of concept, relations, attributes connected in the field of car Network , defined as a five tuple: $\mathrm{O}=(\mathrm{C}, \mathrm{I}, \mathrm{R}, \mathrm{A}, \mathrm{F})$.

Where: $\mathrm{C}$ is the concept set of car networking, is a collection of basic concept car in the car networking, is the result of the abstraction of example, its implication have clear description. I is the collection of instance, is specific instance of concept in concept set. $\mathrm{R}$ is a collection of relationship, including the relations between concept and concept, between association association, between concepts and association, association type can be the mapping that one to one, one to many, many to many. A is a set of attribute, is the description of the concept and feature of relation. $\mathrm{F}$ is a set of axiom, express the constraint rules between these elements.

\section{Research Scope of CAR Networking Ontology}

Vehicle road network relates to two fields the intelligent transportation and networking .Is the overlap that the two large-scale system in highway traffic application domain . So 
in the analysis of vehicle road network knowledge field, It is necessary to consider the intelligence services that be provided in Intelligent Transportation and also consider the interconnection characteristics between car and car, between vehicles and infrastructure. Therefore, the vehicle road network research range can be determined as three sub field: service management field which provide intelligent traffic service, based equipment field which complete the basic functions of the Internet of things, knowledge management field that Implement effectively connecting with infrastructure and services. the field division of the relevant vehicle road network as shown in Figure 1.

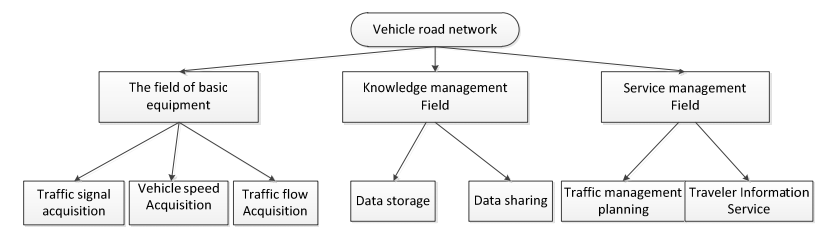

FIGURE I. HIERARCHICAL STRUCTURE OF CAR ROAD NETWORK FIELD.

\section{INTELligent PERCEPTION OF GROUP OF VEHICLE}

Figure 2 is the application hierarchical structure chart of perception layer. In the figure sensing layer of car road network is divided into sensing device layer, equipment network layer, equipment coordination layer. Where, sensing device layer can be abstracted as the sensing component, controlled components, the sensing component includes sensors, RFID, bar code, intelligent detection instrument, controlled components include valve switch, relay, some actuator device that work according to the logic relation , to realize the functions such as signal acquisition, processing, control . Equipment network layer includes various bus such as CAN bus, RS-485 bus, or wireless such as WSN, Bluetooth , WiFi etc, to realize communication connection between the sensing equipment and sensing equipment , between the sensing equipment and gateway of vehicle road network. Equipment coordination layer is gateway of car road network, to realize the unified management of the sensing device, make the sensing layer more real-time and reliable through the real-time scheduling.

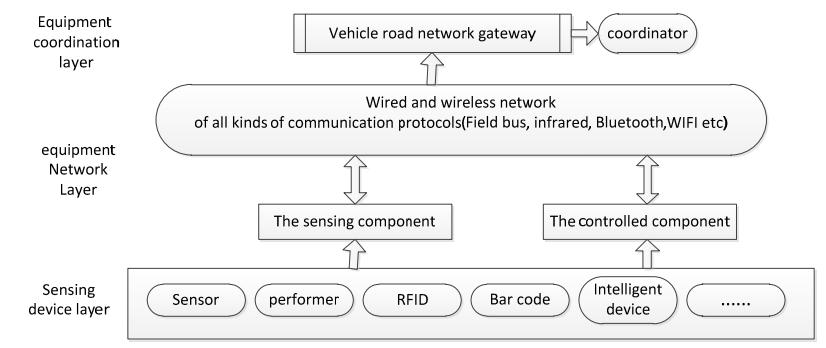

FIGURE II. STRUCTURE DIAGRAM OF CAR ROAD NETWORK SENSING LAYER.

\section{TRAFFic Route Guidance}

The design of function of intelligent traffic route guidance system .

The system has function of the vehicle positioning, map browsing, optimal route guidance. The basic structure of the system is consists of five parts, as shown in Figure 3.

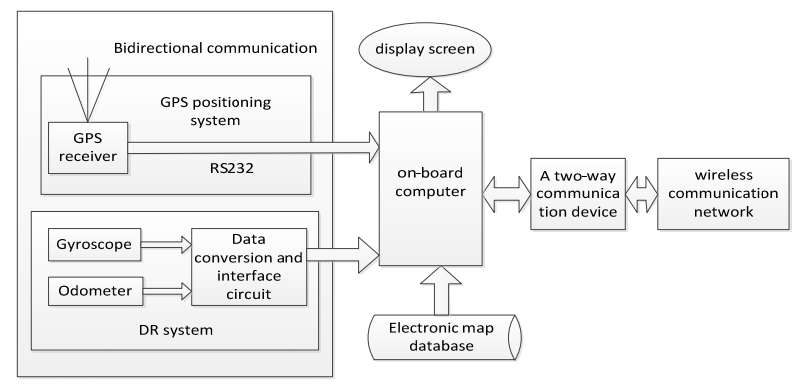

FIGURE III. INTELLIGENT TRAFFIC ROUTE GUIDANCE SYSTEM.

\section{CONCLUSION}

This paper carry on deeply research on semantic modeling, intelligent information perception and traffic route guidance on issues such as road of vehicle road network on the basis of previous studies. To provide guidance for the development of intelligent transportation system of China.

\section{ACKNOWLEDGEMENT}

In this paper, the research was sponsored by the Nature Science Foundation of Zhejiang Province (Project No. Q14F010008) and the Nature Science Foundation of Ningbo City (Project No. 201401A6101039) and the national science and technology support program(Project No. 2012BAF12B11).

\section{REFERENCES}

[1] Saha A K, Johnson D B. Modeling Mobility for Vehicular Ad-Hoc Networks[C].Proc of the 1st ACM International Workshop on Vehicular Ad Hoc Networks,2004.91-92.

[2] B. Gallagher et al. Wireless Communications for Vehicle Saftey: Radio Link Performance and Wirelss Connectivity Methods[J]. IEEE Vehic. Tech. Mag,2006 1(4) 4-24.

[3] C.F. Mecklenbrauker et al. Vehicluar Channel Characterization and Its Implications for Wireless System Design and Performance[C]. Proc. IEEE, 2011.1189-1212.

[4] S.R. Dickey et al. Field Measurements of Vehicle to Roadside Communication Performance[C]. IEEE VTC-Fall, Baltimore(USA), 2007.2179-2183.

[5] J. Hourdakis, P. G. Michalopoulos, and J. Kottommannil. A practical procedure for calibrating microscopic traffic simulation models[J]. Transp. Res. Record, 2003 1852(3) 130-139 . 